\title{
Measuring time in meters
}

\author{
Open Physics Collaboration*†
}

May 26, 2021

\begin{abstract}
This is a microreview of the natural system of units for pedagogical purpose.
\end{abstract}

keywords: natural system of units, special relativity, time, meters

The most updated version of this white paper is available at https://osf.io/4ntwu/download

\section{Introduction}

1. In the International System of Units (SI), the velocity of light in vacuum is, approximately, $3 \cdot 10^{8} \mathrm{~m} / \mathrm{s}[1]$.

2. The Natural System of Units is overwhelmingly useful in Quantum Field Theory calculations [2], whereas the SI is usually used in the context of classical physics.

*All authors with their affiliations appear at the end of this white paper.

†Corresponding author: mplobo@uft.edu.br|Open Physics Collaboration 


\section{What does $c=1$ mean?}

3. Let's consider (2) hereafter.

4. The measurement of time is accomplished by means of the distance that light travels in vacuum [3].

5. 1 second is the time required for light to travel $3 \cdot 10^{8} \mathrm{~m}$.

6. Thus, 1 second in SI is equivalent to $3 \cdot 10^{8} \mathrm{~m}$ in the natural system of units.

7. For short, NSU $=$ Natural System of Units.

8. Let's calculate the light speed in the NSU.

9. Consider that light in vacuum travels the distance $\Delta x=3 \cdot 10^{8} \mathrm{~m}$.

10. Then, $c=\frac{\Delta x}{\Delta t}=\frac{3 \cdot 10^{8} \mathrm{~m}}{\Delta t}$.

11. Recall, from (6), that $\Delta x=3 \cdot 10^{8} \mathrm{~m}$, then $\Delta t=1 \mathrm{~s}=3 \cdot 10^{8} \mathrm{~m}$.

12. Substituting $\Delta t=3 \cdot 10^{8} \mathrm{~m}$ in (10), $c=\frac{3 \cdot 10^{8} \mathrm{~m}}{3 \cdot 10^{8} \mathrm{~m}}=1$.

13. So, in the NSU, $c=\frac{1 \mathrm{~m}}{1 \mathrm{~m}}$, or simply $c=1$.

14. Einstein's equation, $E=m c^{2}$, becomes $E=m$.

\section{Final Remarks}

15. We presented the physical motivation and meaning for $c=1$.

16. The Natural System of Units is developed from a natural phenomenon, that is, the speed of light. 


\section{Open Invitation}

Review, add content, and co-author this white paper $[4,5]$.

Join the Open Mathematics Collaboration.

Send your contribution to mplobo@uft.edu.br.

\section{Open Science}

The latex file for this white paper together with other supplementary files are available in [6].

\section{How to cite this paper?}

https://doi.org/10.31219/osf.io/4ntwu

\section{Acknowledgements}

+ Open Science Framework https://osf .io

+ Zenodo https://zenodo.org

\section{Agreement}

17. All authors agree with [5].

\section{References}

[1] Wikipedia. International System of Units. https://en.wikipedia.org/wiki/International_System_of_Units 
[2] Mandl, Franz, and Graham Shaw. Quantum Field Theory. John Wiley \& Sons, 2010.

[3] Taylor, Edwin F., and John Archibald Wheeler. Spacetime Physics. Macmillan, 1992.

[4] Lobo, Matheus P. "Microarticles." OSF Preprints, 28 Oct. 2019. https://doi.org/10.31219/osf .io/ejrct

[5] Lobo, Matheus P. "Simple Guidelines for Authors: Open Journal of Mathematics and Physics." OSF Preprints, 15 Nov. 2019.

https://doi.org/10.31219/osf.io/fk836

[6] Lobo, Matheus P. "Open Journal of Mathematics and Physics (OJMP)." OSF, 21 Apr. 2020. https://osf .io/6hzyp/files

\section{The Open Physics Collaboration}

Matheus Pereira Lobo (lead author, mplobo@uft.edu.br) ${ }^{1}$

https://orcid.org/0000-0003-4554-1372

Caio Matheus Fontinele dos Santos

João Marcos Costa da Silva

Eduardo Barros Oliveira

${ }^{1}$ Federal University of Tocantins (Brazil) 